\title{
DECISIONS
}

\section{A 65-year-old HIV-positive man with acute diarrhea}

\author{
Philip W. Lam MD, Paul E. Bunce MD
}

A 65-year-old man with chronic HIV infection presents with a one-week history of diarrhea. He describes six to eight loose bowel movements daily. His most recent CD4 count is $0.22 \times 10^{9} / \mathrm{L}$, with an undetectable viral load. His current antiretroviral (ARV) regimen consists of ritonavirboosted atazanavir, tenofovir and emtricitabine.

\section{What are the key components of history to obtain for this patient?}

In addition to onset and duration of the diarrhea, details such as volume and frequency of stools, nocturnal symptoms, abdominal pain, fever and weight loss may provide clues to the underlying cause. ${ }^{1}$ Sexual activities, food and water exposures, recent travel and antibiotic use should also be assessed. In any patient with HIV, the history should also include the duration of HIV infection, past HIV-related complications, current and past ARV regimens (and adherence), and most recent T-lymphocyte CD4 count, as these details can provide insight into the susceptibility to opportunistic pathogens. ${ }^{1}$

\section{What is the differential diagnosis of acute diarrhea in this patient?}

Potential complications of HIV infection can be divided into HIV-related and HIV-unrelated causes (i.e., conditions that commonly occur in HIV-negative individuals) (Figure 1). Causes related to HIV occur almost exclusively in immunocompromised or HIV-positive patients, and include opportunistic infections or malignant diseases, ARV-related effects and HIV infection itself.

In patients with diarrhea and a CD4 count below $0.10 \times 10^{9} / \mathrm{L}$, potential opportunistic infections include Cryptosporidium species and microsporidia. ${ }^{1}$ Below a CD4 count of $0.05 \times 10^{9} / \mathrm{L}$, patients are at risk of acquiring cytomegalovirus (CMV) colitis and disseminated Mycobacterium avium complex disease. ${ }^{1}$ Opportunistic malignant diseases such as non-Hodgkin lymphoma and Kaposi sarcoma can present with diarrhea when the gastrointestinal tract is involved.

Diarrhea caused by ARV agents is common. Incidence of diarrhea is highest with protease inhibitors (13.6\%), followed by nucleoside reverse transcriptase inhibitors $(10.0 \%)$ and non-nucleoside reverse transcriptase inhibitors $(2.2 \%) .^{2}$ The incidence of diarrhea also varies among ritonavir-boosted protease inhibitors, ranging from $2 \%$ (ritonavir-boosted atazanavir) to $11 \%$ (ritonavir-boosted lopinavir). ${ }^{2}$

Diarrhea caused by HIV itself (known as HIV enteropathy) should be considered when other causes have been excluded.

Causes unrelated to HIV, such as infectious (e.g., Escherichia coli, Campylobacter, Shigella, Clostridium difficile, viral), drug-induced (e.g., laxatives), dietary and immunologic causes, remain important considerations as well. A broader differential diagnosis exists in patients who present with chronic diarrhea, and includes inflammatory bowel disease, irritable bowel syndrome and gluten-sensitive enteropathy.

\section{What initial investigations should be ordered?}

After a clinical assessment of volume status and stability, serum electrolytes and creatinine should be measured to assess for metabolic or renal abnormalities secondary to volume depletion. Depending on the severity of diarrhea, and the presence of

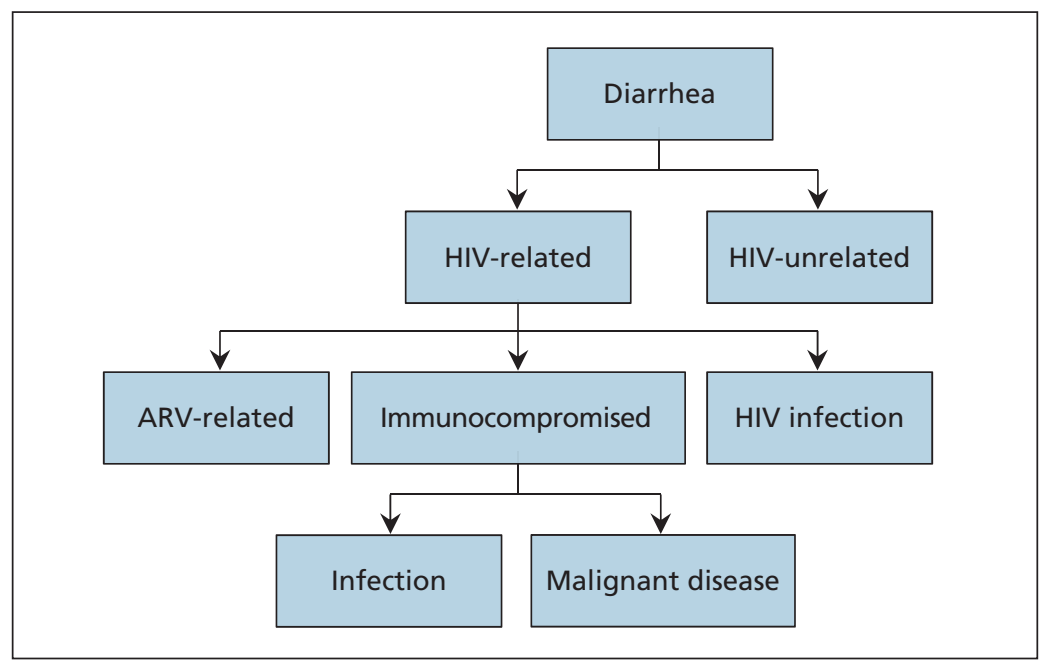

Figure 1: Approach to the diagnosis of diarrhea in a patient with HIV infection. ARV = antiretroviral therapy.
Competing interests: None declared.

This article has been peer reviewed.

Correspondence to: Paul E. Bunce, paul.bunce@uhn.ca /cmaj.150183
CMAJ 2015. DOI:10.1503 
metabolic derangements, admission to hospital for intravenous fluid replacement may be necessary.

Recommendations from the Panel on Antiretroviral Guidelines for Adults and Adolescents suggest that initial investigations include a CD4 count if previous results from blood tests are unavailable or tests were done more than three months previously. ${ }^{3}$ A stool sample should be sent for bacterial culture and to test for ova, cysts and parasites; the latter test should be run on samples collected on at least two separate days. ${ }^{4}$ Specimen requisitions should identify the patient as HIV-positive or request acid-fast (to detect Cryptosporidium, Isospora and Cyclospora) and trichrome staining (to detect microsporidia) because these are not routinely performed. ${ }^{1} \mathrm{~A}$ stool sample should also be collected to test for Clostridium difficile. ${ }^{1}$ Depending on the extent of depletion of CD4 lymphocytes and the clinical history, additional tests to be considered include blood cultures, as well as blood and stool cultures for acid-fast bacilli.

If symptoms persist despite negative stool investigations, a referral to a gastroenterologist should be made for consideration of endoscopy. ${ }^{1}$ Direct visualization of the gastrointestinal mucosa and histopathology from biopsy samples can improve diagnostic yield. In addition, the diagnosis of CMV colitis is based on histopathologic findings, because it can occur in the absence of detectable CMV viremia. ${ }^{5}$ Current guidelines based on expert opinion suggest flexible sigmoidoscopy or colonoscopy when laboratory investigations are nondiagnostic, with consideration for upper endoscopy if symptoms persist. ${ }^{6}$

\section{Should ARV be withheld at this time?}

When considering temporary discontinuation of ARV regimens, clinicians must weigh the likelihood of ARV toxicity, severity of illness and potential harm if an offending agent is continued against the potential harms of treatment interruption. Factors suggesting ARV-related diarrhea include an ARV regimen known to cause diarrhea and a temporal relation between diarrhea and ARV initiation. Current guidelines recommend shortterm interruption of ARV only when a patient experiences severe or life-threatening toxicity or cannot take oral medications. ${ }^{3}$ If a decision is made to interrupt treatment, the entire ARV regimen should be withheld. Long-term interruption of ARV therapy is not recommended. ${ }^{3}$ Management of ARV-associated diarrhea should be carried out in consultation with a clinician who has experience managing the treatment of patients with HIV.

\section{Case revisited}

On further history, no unique exposures were identified. Results of routine laboratory investigations were normal. Stool cultures isolated Shigella species. Because of his immunocompromise, and based on susceptibility testing, the patient received ciprofloxacin $500 \mathrm{mg}$ orally twice daily for seven days, and his symptoms resolved. The patient was also counselled on the risks of Shigella transmission with sexual activity, and the importance of safe sexual practice and proper hand hygiene.

\section{References}

1. Guidelines for the prevention and treatment of opportunistic infections in HIV-infected adults and adolescents: recommendations from the Centers for Disease Control and Prevention, the National Institutes of Health, and the HIV Medicine Association of the Infectious Diseases Society of America. Rockville (MD): Panel on Opportunistic Infections in HIV-Infected Adults and Adolescents. Available: http://aidsinfo.nih.gov/contentfiles/ lvguidelines/adult_oi.pdf (accessed 2015 Feb. 5).

2. Clay PG, Crutchley RD. Noninfectious diarrhea in HIV seropositive individuals: a review of prevalence rates, etiology, and management in the era of combination antiretroviral therapy. Infect Dis Ther 2014 Nov. 12. [Epub ahead of print].

3. Panel on Antiretroviral Guidelines for Adults and Adolescents. Guidelines for the use of antiretroviral agents in HIV-1-infected adults and adolescents. Rockville (MD): US Department of Health and Human Services. Available: http://aidsinfo.nih.gov/ contentfiles/lvguidelines/AdultandAdolescentGL.pdf (accessed 2015 Feb. 5).

4. Cartwright CP. Utility of multiple-stool-specimen ova and parasite examinations in a high-prevalence setting. J Clin Microbiol 1999;37:2408-11.

5. Dieterich DT, Rahmin M. Cytomegalovirus colitis in AIDS: presentation in 44 patients and a review of the literature. $J$ Acquir Immune Defic Syndr 1991;4(Suppl 1):S29-35.

6. Shen B, Khan K, Ikenberry SO, et al.; ASGE Standards of Practice Committee. The role of endoscopy in the management of patients with diarrhea. Gastrointest Endosc 2010;71:887-92.

Affiliations: Department of Medicine (Lam, Bunce), University of Toronto; Division of Infectious Diseases (Bunce), University Health Network, Toronto, Ont.

Contributors: Both authors contributed to writing the manuscript, approved the final version to be published and agreed to act as guarantors of the work.

Decisions is a series that focuses on practical evidence-based approaches to common presentations in primary care. The articles address key decisions that a clinician may encounter during initial assessment. The information presented can usually be covered in a typical primary care appointment. Articles should be no longer than 650 words, may include one box, figure or table and should begin with a very brief description ( 75 words or less) of the clinical situation. The decisions addressed should be presented in the form of questions. A box providing helpful resources for the patient or physician is encouraged. 